\title{
To Compare the Effects of Footwear in Young Female's Postural Balance in Prolonged Standing and Sitting Job
}

\author{
Anandita Sharma', Yamini Sharma², Megha Yadav², \\ Mangalam Kumari², Jyoti Sharma ${ }^{2}$, Sakshi Arora ${ }^{2}$, Akshay Anand ${ }^{3}$

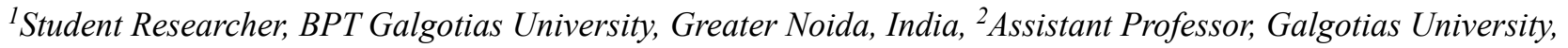 \\ Greater Noida, India, ${ }^{3}$ Assistant Professor, Manav Rachna International University, Faridabad, India
}

\begin{abstract}
Background: Footwear has an essential role in raising well-being of any person. Many females have wear heel or flat footwear on their daily basis as if they are working or not. Footwear creates discomfort which leads to pain in foot, and results in alteration of balance. Most of the working places requires prolonged period of standing which is possibly associated with musculoskeletal disorders, likely affects our health and acute trauma from the working places. Prolonged sitting and standing can directly affect the weight-bearing joints majorly the hip, knee and ankle joint which in turn leads to deformities and effects balance. Changes in this composite system results in the risk of falls and loss of stability.

Objective: The objective of the study was to identify the effects of footwear in young female's postural balance in prolonged standing and sitting job.

Materials and Method: 100 healthy females were taken for the study age 18-35 years but only 60 were selected for the study. The subject had to perform Rhomberg test and tandem walk for balance assessment.

Result: On analysis, it was found that in rhomberg balance test, $73.33 \%$ population were positive with the test. $8.33 \%$ population were positive with the eyes opened and $65 \%$ population were positive with the eyes closed and in tandem walk, $31.66 \%$ population is positive who were unable to complete the test.

Conclusion: In conclusion, the balance tests shows that, wearing footwear for prolonged hours of standing and sitting job,states that the balance and postural control worsening which is associated with decrease in postural stability and increase in the risk of falls.. According to the study balance is more effected in prolonged sitting than in prolonged standing.
\end{abstract}

Keywords: Balance, postural control.

\section{Introduction}

There are many different professions in our country in which females are working. Some have sitting jobs (co-operative jobs,) and other have standing jobs (female

\footnotetext{
Corresponding Author:

Akshay Anand

Assistant Professor, Manav Rachna International

University, Faridabad

e-mail: akshayaanad.anand25@gmail.com
}

guards, CRPF females, female traffic police job etc). During long hours of job, women prefer footwear which are more comfortable in standing as well as in sitting. Footwear are considered by the women as an essential part of their regular outfit. There are different types of fancy footwear females have wear to appear good and this also enhances their personality.

With generality of $39 \%$ noticed in institutional settings and $78 \%$ of generality noticed in clinical settings which showed a massive ratio of female population and with this usage of different heels and flat footwear leading to alterations in the person postural balance. 
There was a survey conducted by the hotter shoes which suggested that to look attractive women goes to such extent that they wear different heels which extremely very high which are leading to problems such as sore foot and tired foot because of wearing them whole day. ${ }^{1}$ Murphy et al. had recommended that footwear were the key component in the building of human posture. ${ }^{2}$

Footwear has an essential role in raising well-being of any person. ${ }^{3}$ As footwear passes the sensory information to the foot, and a person can control the postural stability through the touch and proprioception. ${ }^{4,5}$ Footwear creates discomfort which leads to pain in foot, and results in alteration of balance.

Balance can be defined as a situation in which one's body dynamics are proportionate just to prevent the person's body from falling and also the ability to uphold, attain and reinstate the person's body center of mass (COM) respective to the base of support (BOS). Maintaining balance is acknowledged as keeping the center of gravity (COG) in BOS defined by the feet. ${ }^{7}$ Balance is sustained through dynamic combination of internal and external. ${ }^{8}$ Functional activity and motion is necessary for balance. Functional balance is capability of an individual to maintain a position and posture during any functional motion. ${ }^{2}$

Postural control or balance can be defined statically as the ability to maintain a base of support with minimal movement and dynamically as the ability to perform a task while maintaining a stable position. ${ }^{10}$

The aim of the study is to determine the effects of footwear on young female's postural balance in prolonged standing and sitting job. Many researches have been done on effects of footwear on balance but this research will leads to explain the how footwear effects the balance in prolonged standing and sitting job. For static balance test, rhomberg berg test has been done and for dynamic balance test, tandem walk has been done.

Prolonged period of working is defined as standing and sitting for 50 percent of the working day or more of it. ${ }^{11}$ Most of the working places requires prolonged period of standing which is possibly associated with musculoskeletal disorders,likely affects our health and acute trauma from the working places. Most of the interviews disclosed excessive proportion of musculoskeletal discomfort and environmental demands and other chronic problems. ${ }^{12}$ Neck and trunk flexion in prolonged standing in day to day life is the threat which causing skeletal disorders and it can lead to various problems in legs and feet such as discomfort, fatigue and pain in neck, lower back, hip, spinal compression, chronic venous insufficiency. It also increases risk of heart attack, swelling due to impaired circulation and decreases the oxygenation and nutrients supply leads to muscle fatigue. ${ }^{1}$

Static postures such as prolonged sitting and prolonged standing increase physical and mental exertion on the body and these exertions increases the force on the musculoskeletal system. ${ }^{13}$ Disuse of muscles by prolongedsitting can cause weakness of muscles. Prolonged sitting can lead to ischemia, apoptosis (programmed cell death), it may directly affect the musculoskeletal system ${ }^{13}$ like pain in legs, ${ }^{16}$ chronic venous insufficiency, ${ }^{18}$ spinal compression, ${ }^{15}$ and impaired circulation which in result causes swelling of the lower extremities. ${ }^{13,14}$. During standing in one position or maintaining the erect posture without an ease of walking rhythmically, affects the blood circulation and compromised body fluids. ${ }^{13}$ This causes swelling and pooling in lower extremities. ${ }^{13,14}$

Prolonged sitting and standing can directly affect the weight-bearing joints majorly the hip, knee and the ankle joint which in turn leads to deformities and effects balance. Changes in this composite system results in the risk of falls and loss of stability. ${ }^{3}$ In maintaining the balance of the body which may be deformed both by intrinsic forces (body movement), and by the extrinsic forces (the forces which is acting on the body in a certain environment). ${ }^{3}$

There are many more researches based on balance but there is no research had been done on the effects of footwear on young female's postural balance in prolonged standing and sitting job. This research will provide the intra subject reliability of balance measurements in young females.

\section{Material and Method}

Study Area: This study was carried out from the various working places.

Study Design: This was an observational study.

Study Population: The study population consisted of 100 young females.

\section{Study criteria:}


Inclusion: Healthy female's (18-35years).

Exclusion: Any diagnosed history of recent muculoskeletal trauma, congenital anomalities, joint pain, neurological disorders.

\section{Procedure:}

1. Rhomberg test: In this test, the subject was asked to stand with the feet together and arms hanging alongside. The subject is instructed to stand for 30 seconds with eyes open and the postural sway was observed. Then we asked the subject to close her eyes The test is positive if sway or imbalance was there.

2. Tandem walk: The subject is instructed to fold their arms across their chest walk by placing one foot directly in front of the other, like heel-to-toe walking. Walking as fast as they could for 30 steps. $^{17}$ The test is positive if the subject cannot walk in heel

to toe position or if the subject is disbalanced during the test.

\section{Result}

The total subjects taken for this study were 100 but only 60 were fit for the study and 40 subjects were excluded on the basis of exclusion criteria.

This is an observational study and there is some inclusion and exclusion criteria for the accuracy of the data which was collected from the subjects. The Balance tests which have been done to identify the static and the dynamic balance. Rhomberg test have been done with eyes open and close to identify the static balance. In Rhomberg test, $73.33 \%$ population were positive with the test. $8.33 \%$ population were positive with the eyes opened and $65 \%$ population were positive with the eyes closed. (Table 1).Tandem walk were used to observe the dynamic balance, $31.66 \%$ population is positive who were unable to complete the test. (Table 2).

Data:

Table 1: Rhomberg Test

\begin{tabular}{|l|c|c|c|}
\hline Parameters & No. of subjects & Percentage (\%) & Mean \pm Standard Deviation \\
\hline 1a. Eyes open & 55 & $91.66 \%$ & \\
\hline No Sway & 5 & $8.33 \%$ & $0.9166 \pm 0.2763$ \\
Sway & 21 & $35 \%$ & \\
\hline 1b. Eyes closed & 39 & $65 \%$ & $0.35 \pm 0.4769$ \\
\hline No Sway &
\end{tabular}

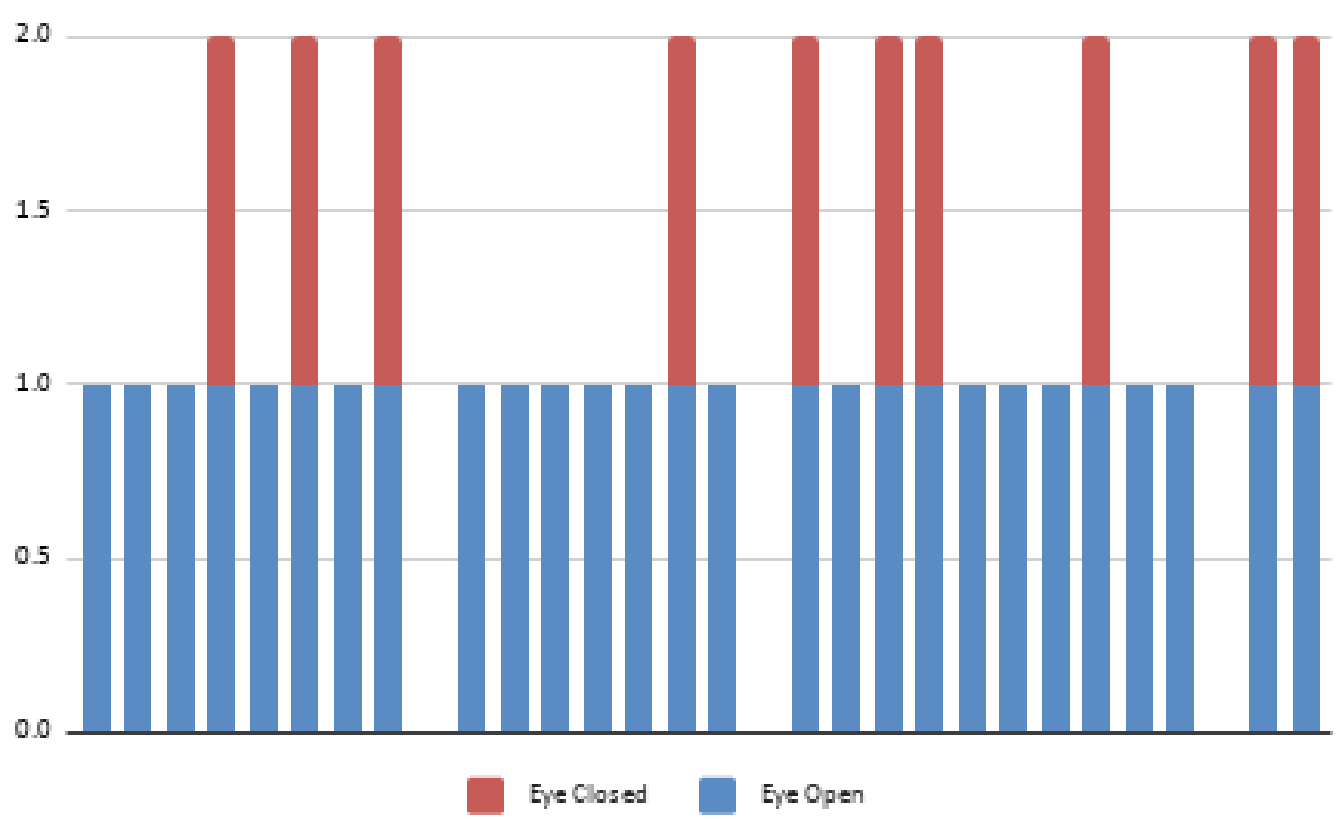

Graph 1: Showing data of eye closed and eye open subjects. 
The graph showed the percentage of the subjects sway or no sway during Rhomberg's eyes open and close test.

Table 2: Tandem walk

\begin{tabular}{|l|c|c|c|}
\hline Parameters & No. of subjects & Percentage (\%) & Mean \pm Standard Deviation \\
\hline Positive test & 19 & $31.66 \%$ & $0.6833 \pm 0.4561$ \\
Negative test & 41 & $68.33 \%$ & \\
\hline
\end{tabular}

Table 2 This graphs showed the relation between the age and the test performed by the subjects.

\section{Discussion}

In this research, we have examined the effects of footwear on young female's postural balance in prolonged standing and sitting job by the balance test examination.

The static and dynamic balance is affected by the property of footwear which includes their materials, stiffness, and soles thickness, collar height and the coefficient of friction of the outer sole of the footwear. ${ }^{19}$ Several earlier studies have been studied about relation of balance and The ability of static and dynamic balance due to wearing high heels and causes muscle fatigue. ${ }^{20}$ Heterogeneity of footwear in young females and the females in their 20 s prefer to wear low heeled casual footwear in spite of any motive or wants.. ${ }^{21}$ Another study examined that duration of wearing high heels in young adults revealed that the muscles such as rectus femoris and tibialis anterior did not indicate any such significant differences in lower extremities muscle activity and in static balance. ${ }^{6}$

Most of the studies have accomplished the effects of footwear on balance. Thus, the basis of this research was to the examined the effects of footwear in a specifically young aged female's postural balance. For prolonged standing and sitting causes musculoskeletal problems and other related problems like pain in legs, spinal

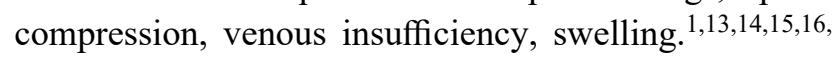
People should deliberate in their daily life about the types of shoes and the heel height. ${ }^{2}$ The balance tests which have been done in this research studies states that the alteration in balance of females due to footwear is affected. The subjects when performing the tests were more dis-balanced in eyes closed task as compared in eyes open. And if we compare the test between the tandem walk and rhomberg's eyes open and close tests, the subjects having more difficulty in performing the rhomberg's eyes closed test. According to the results, females have to wear footwear according to their comfort ability and during prolonged hours of jobs in sitting and standing dynamics, they need to relax her foot in every hour in order to keep their foot relaxed and it will prevent from swelling, leg pain and other causes which we have discussed in our research study.

\section{Limitation of Study:}

- Less number of subjects.

- Less number of tests is used to assess balance.

- Heel height.

- Type of heels.

- We do not know that the subjects involve in any sports activities.

- We did not compare the subject's arches whether they are flat foot, pes cavus or normal.

\section{Future Scope:}

- It will help in the prevention, evaluation and treatment of primary and secondary structural damage in foot, and other musculoskeletal conditions.

- We only took the young women but with the inclusion of women of middle and old age, the generalisability will increase and we can have a better result.

- We had only compare the result between females, for further studies use comparative effects in males and females in prolonged working hours.

\section{Conclusion}

In conclusion, this study examined the effects of footwear in young females postural balance in prolonged standing and sitting job. The result showed the significant differences in rhomberg test. The subjects having difficulty during eyes closed test than in eyes open and in tandem walk test showed that the subjects 
having difficulty in heel to toe walking because of having trouble in maintaining balance.

In the study the balance tests shows that, wearing footwear for prolonged hours of job, states that the balance and postural control worsening which is associated with decreases in the postural stability and increases in the risk of falls. According to the study, balance is more effected in prolonged sitting than in prolonged standing.

Conflict of Interest: None

Ethical Clearance: Institutional ethical committee

Sources of Funding: Self

\section{References}

1. Sema Ozandaç Polat, Ahmet Hilmi Yücel "the negative effects of high heels on the biomechanics and somatometry on the lower extrimities." 2018

2. Mi-Kyoung Kim, Byung-Sun Kong, Kyung-Tae Yoo "The effect of shoe type on static and dynamic balance during treadmill walking in young healthy women"2017

3. Barton CJ, Bonanno D, Menz HB. "Development and evaluation of a tool for the assessment of footwear characteristics"2009.

4. Hijmans JM, Geertzen JH, Dijkstra PU, Postema K. " A systematic review of the effects of shoes and other ankle or foot appliances on balance in older people and people with peripheral nervous system disorders" 2007

5. Perry SD, Radtke A, Goodwin CR "Influence of footwear midsole material hardness on dynamic balance control during unexpected gait termination"2007

6. Lee MH, Chang JS, Lee SY, et al.: The effects of high-heeled shoes on static balance and EMG activity of lower extremity muscles for young women"2009

7. Peterka RJ. " Sensorimotor integration in human postural control. Journal of neurophysiology" 2002

8. Yaggie JA, McGregor SJ. "Effects of isokinetic ankle fatigue on the maintenance of balance and postural limits"Arch Phys Med Rehabil, 2002.
9. Kisner C, Colby LA, Borstad J. Therapeutic exercise: foundations and techniques. Fa Davis; 2017

10. Winter DA, Patla AE, Frank JS. Assessment of balance control in humans. Med Prog Technol. 1990

11. Tomei F, Baccolo TP, Tomao E, Palmi S, Rosati MV. "Chronic venous disorders and occupation"1999

12. Jennifer Anderson, Anita E. Williams \& Christopher Nester " An explorative qualitative study to determine the footwear needs of workers in standing environments"2016

13. Nancy L. Hughes, Audrey Nelson, Mary W. Matz, John Lloyd." Solutions for prolonged standing in perioperative setting" 2011

14. Canadian Centre for Occupational Health \& Safety "Working in a standing position-basic information" 2010 .

15. Beynon C, Reilly T."Spinal shrinkage during a seated break and standing break during simulated nursing tasks"2001

16. Cook J, Branch TP, Baranowski TJ, Hutton WC."The effect of surgical floor mats in prolonged standing: an EMG study of the lumbar paraspinal and anterior tibialis muscles"1993

17. Chantanachai T, Pichaiyongwongdee $\mathrm{S}$, Jalayondeja C Journal of the Medical Association of Thailand=Chotmaihet Thangphaet "Fall prediction in thaielderly with timed up and go and tandem walk test: a cross sectional study" 2014

18. Redfern MS. Cham R. "The influence of flooring on standing comfort and fatigue" 2000.

19. Boyas S, McGown C, Lajoie Y, et al. "Changes in posture associated with postural control impairment induced by ankle plantarflexor fatigue during unipedal quiet standing”2013

20. Kim YJ,Koo JW, Oh DW "Influence of shoe heel height and muscle fatigue on static and dynamic balance in healthy young women".2013

21. Kim SN: (A) Survey on shoes wearing and a proposal regarding shoes size and shape improvement: focused on women in their teens and twenties. Ewha Womans University Graduate School, Master's thesis, 2001. 